This is an electronic reprint of the original article. This reprint may differ from the original in pagination and typographic detail.

Author(s): Arstila, Kai; Julin, Jaakko; Laitinen, Mikko; Aalto, Jarkko; Konu, Timo; Kärkkäinen, Samuli; Rahkonen, Samuli; Raunio, Miika; Itkonen, Jonne; Santanen, Jukka-Pekka; Tuovinen, Tero; Sajavaara, Timo

Title: $\quad$ Potku - New analysis software for heavy ion elastic recoil detection analysis

Year: $\quad 2014$

Version:

Please cite the original version:

Arstila, K., Julin, J., Laitinen, M., Aalto, J., Konu, T., Kärkkäinen, S., Rahkonen, S., Raunio, M., Itkonen, J., Santanen, J.-P., Tuovinen, T., \& Sajavaara, T. (2014). Potku New analysis software for heavy ion elastic recoil detection analysis. Nuclear Instruments and Methods in Physics Research, Section B: Beam Interactions with Materials and Atoms, 331(July), 34-41. https://doi.org/10.1016/j.nimb.2014.02.016

All material supplied via JYX is protected by copyright and other intellectual property rights, and duplication or sale of all or part of any of the repository collections is not permitted, except that material may be duplicated by you for your research use or educational purposes in electronic or print form. You must obtain permission for any other use. Electronic or print copies may not be offered, whether for sale or otherwise to anyone who is not an authorised user. 


\title{
Potku - New Analysis Software for Heavy Ion Elastic Recoil Detection Analysis
}

\author{
K. Arstilaa,,* J. Julin ${ }^{\mathrm{a}}$, M.I. Laitinen ${ }^{\mathrm{a}}$, J. Aalto ${ }^{\mathrm{b}}$, T. Konu ${ }^{\mathrm{b}}$, S. Kärkkäinen ${ }^{\mathrm{b}}$, S. Rahkonen ${ }^{\mathrm{b}}$, M. Raunio ${ }^{\mathrm{b}}$, J. Itkonen ${ }^{\mathrm{b}}$, J.-P. Santanen ${ }^{\mathrm{b}}$, \\ T. Tuovinen ${ }^{\mathrm{b}}$, T. Sajavaara ${ }^{\mathrm{a}}$ \\ ${ }^{a}$ Department of Physics, P.O.Box 35, FI-40014 University of Jyväskylä, Finland \\ ${ }^{b}$ Department of Mathematical Information Technology, P.O.Box 35, FI-40014 University of Jyväskylä, Finland
}

\begin{abstract}
Time-of-flight elastic recoil detection (ToF-ERD) analysis software has been developed. The software combines a Python-language graphical front-end with a $\mathrm{C}$ code computing back-end in a user-friendly way. The software uses a list of coincident time-offlight-energy (ToF-E) events as an input. The ToF calibration can be determined with a simple graphical procedure. The graphical interface allows the user to select different elements and isotopes from a ToF-E histogram and to convert the selections to individual elemental energy and depth profiles. The resulting sample composition can be presented as relative or absolute concentrations by integrating the depth profiles over user-defined ranges. Beam induced composition changes can be studied by displaying the eventbased data in fractions relative to the substrate reference data. Optional angular input data allows for kinematic correction of the depth profiles. This open source software is distributed under the GPL license for Linux, Mac, and Windows environments.
\end{abstract}

Keywords: ion beam analysis, elastic recoil detection, ERD, TOF-ERD, graphical open source software

\section{Introduction}

In ion beam analysis (IBA) measurements the availability of reliable and well documented analysis software is of key importance for producing credible and traceable results with a technique in use. For Rutherford backscattering spectroscopy (RBS) and particle-induced x-ray emission spectroscopy (PIXE), several well established software products, such as SIMNRA [1], WinNDF [2] and GUPIX [3], are available. For heavy ion elastic recoil detection analysis (HI-ERDA) some analysis software has been proposed [4], but most of the laboratories are still using in-house developed or non-public software which generally cannot offer a traceable analysis path.

To answer the need of a publicly available user friendly analysis software for HI-ERDA, a project was launched at the Physics Department of University of Jyväskylä. The software Potku (in Finnish kick or recoil) was started as a student software project in collaboration with the materials analysis group at the Physics department and the Department of Mathematical Information Technology.

The graphical interface of Potku is realized using Python programming language and the underlying computationally intensive calculations are performed by $\mathrm{C}$ language routines. The software is distributed ${ }^{1}$ under the GNU General Public License and further development is carried out as a project in GitHub ${ }^{2}$.

\footnotetext{
${ }^{*}$ Corresponding author, email: kai.arstila@jyu.fi

${ }^{1} \mathrm{~A}$ stable version of the software is available from http://www.jyu.fi/accelerator/potku

${ }^{2} \mathrm{http}: / /$ github.com/jaakkojulin/potku
}

\section{Physics background of the software}

\subsection{Stopping force calculation}

The determination of stopping force values of a given sample for specific energetic ions is the key feature of any ion beam analysis software. The calculated elemental depth profiles are entirely dependent on the stopping force values. Usually the stopping values are computed for each required iontarget-element pair $\left(\mathrm{Z}_{1}, \mathrm{Z}_{2}\right)$ using an external subroutine, such as the one provided by SRIM software [5, 6], or the stopping values are interpolated from tabulated files. The total stopping force of the material is usually calculated by adding the elemental $\mathrm{Z}_{1}, \mathrm{Z}_{2}$ contributions using the Bragg's rule [7].

The use of pre-compiled libraries generally restricts the choice of the software architecture and the use of individual tabulated stopping files is generally only feasible for RBS analysis where required stopping force values are often limited to one ion-target-material pair. In typical ERD measurements the number of required stopping force values for different $\mathrm{Z}_{1}, \mathrm{Z}_{2}$ pairs can be very large. For instance, in this work we used as an analysis example measurements from thin (50-100 nm) films of LiMnO on silicon substrates. The ToF-ERD measurements were performed using ${ }^{35} \mathrm{Cl}$ incident beam and the films contained impurities of hydrogen, carbon, and fluorine. Thus, a total of $56 \mathrm{Z}_{1}, \mathrm{Z}_{2}$ pairs were required for the stopping force calculations. While generic parametrizations can be used to calculate all these combinations, often there are recent data available for a single $\mathrm{Z}_{1}, \mathrm{Z}_{2}$ pair, like $\mathrm{Cl}$ in $\mathrm{Si}$, or the user might want to try correction factors for certain stopping values.

To achieve full architecture indepence and maximum flexibility for stopping force calculations we have developed a software library gsto as part of this work. The gsto library is in- 
dependent of Potku software and can be used in any software where stopping values are needed.

In the gsto library all the stopping values are interpolated from tabulated values given as ascii files. A top-level command file defines the precedence of the files. The stopping values for a certain $\mathrm{Z}_{1}, \mathrm{Z}_{2}$ pair are extracted from the first file containing data for the specific pair. The last file in the command file list serves as a fall-back ensured to contain stopping data for all the stable elements in all elemental target materials. The stopping values can be entered in different units defined in the headers of the files.

As an example, when a user wants to change the stopping values for one $Z_{1}, Z_{2}$ pair in the analysis, he only needs to give one ascii file with the new stopping values, and place the name of this file as first in the top-level command file. Testing completely different stopping models is similarly straightforward by changing the main fall-back file containing the stopping values for all elements. The size of such a file is typically only around $10 \mathrm{MB}$ if each $Z_{1}, Z_{2}$ pair contains stopping values at 100 energy points in a logarithmic scale.

\subsection{Depth profile calculation}

The separation of every sample element in a ToF-ERD measurement allows for determination of sample composition as a function of depth without any prior knowledge of the sample structure [8]. In this iterative procedure the depth profile calculation is started by assuming a homogeneous sample composition obtained by correcting the total yield of each element with the corresponding scattering cross section (screening corrections by Andersen [9] or L'Ecuyer [10] can be selected). Stopping forces for each recoil ion are then calculated for this initial sample composition, and the original depth for each detected recoiled or scattered particle is calculated backwards using the detected energy, stopping forces, and the known measurement geometry. The resulting depth distributions of the detected particles are again used to create a more accurate estimate of the sample composition and its stopping conditions. The calculation of the originating depths of particles and the stopping forces is repeated iteratively until the composition doesn't change significantly. When the sum of the individual elemental concentrations is scaled to the full 100\% (assuming that all elements were detected and recognized) it becomes possible to integrate absolute concentrations over the depth of the thin film.

It should be noted that the iterative depth profile calculation procedure is not limited to the use of recoils as detected particles. The scattered incident ions can be used as well, as long as their origin, ie. corresponding recoiling target atom, can be unambiguously determined. This is often the case in ToF-ERD measurements where samples contains only one heavy mass (heavier than Si substrate) target element, like in LiMnO measured with $\mathrm{Cl}$ ion beam. In this case all the observed high energy indicent ions originate from scatterings with heavy target atoms, like $\mathrm{Mn}$ in the case of LiMnO. As long as the separation can be made, the iterative procedure can be used not only for ERD but for RBS as well.
The event-by-event list-mode calculation requires more computation power than an approach where intermediate elemental energy spectra are generated and used in the calculations. However, the list mode approach allows for more flexibility in advanced calculations. For instance, position information can be included for each event and the corrections can be calculated to compensate the angular dependent kinematics, the angular dependent cross sections, and the path length variation (see the section 3.6). In the event-based data treatment the contributions affecting to the detection probability of the particle, such as the detection efficiency, angular dependent cross section, isotopic effects, and beam-induced damage, can be easily taken into account by using non-unity statistical weights for events. The final depth profiles are then generated by integrating these statistical weights instead of just number of events. The information on the original number of events should, however, be preserved throughout the calculation, since it can be used to evaluate the statistical errors in the resulting composition values.

\section{Key features of the software}

\subsection{Importing and displaying data}

Potku combines a set of measurements under a project. Within a project the measurement settings and the calibration parameters are identical for each measurement unless the user defines measurement-specific parameters.

Typically a project is created for a series of measurements for similar samples using similar measurement conditions. In this demonstration of Potku software we use a project of three measurements for different samples of LiMnO films grown on silicon. Each measurement consist of more than 2 million events. The main window with a measurement project for these three LiMnO samples can be seen in Fig. 1

The measurement data is read in ascii format, where coincidence time-of-flight-energy values are given as pairs of channel numbers. An optional third column can be used to present position data in the form of angular deflection from the center of the detector. Thus, a typical input file is a two or three-column list of numbers consisting of hundreds-of-thousands to several millions of lines.

For importing tool specific data formats Potku software currently offers two options, one for Jyväskylä Pelletron evnt format and another one for commonly used Fast ComTec system Ist format. Importing from other data formats can be easily implemented.

In the evnt format each energy detector or ToF detector event is represented by an ADC number, a channel number and a time stamp value. The import dialog allows user to set graphically the coincidence window according to the distribution of the events in the time axis. Precise setting of the timing window can significantly reduce the background in the ToF-E histogram. As a result evnt import function generates an ascii data file which can be read using the standard data read dialog of Potku. Similarly, importing a binary lst file generates the corresponding ascii file.

After reading the data Potku displays a 2D TOF-E histogram. Normally the ToF channel is given as $\mathrm{x}$ axis and the energy 


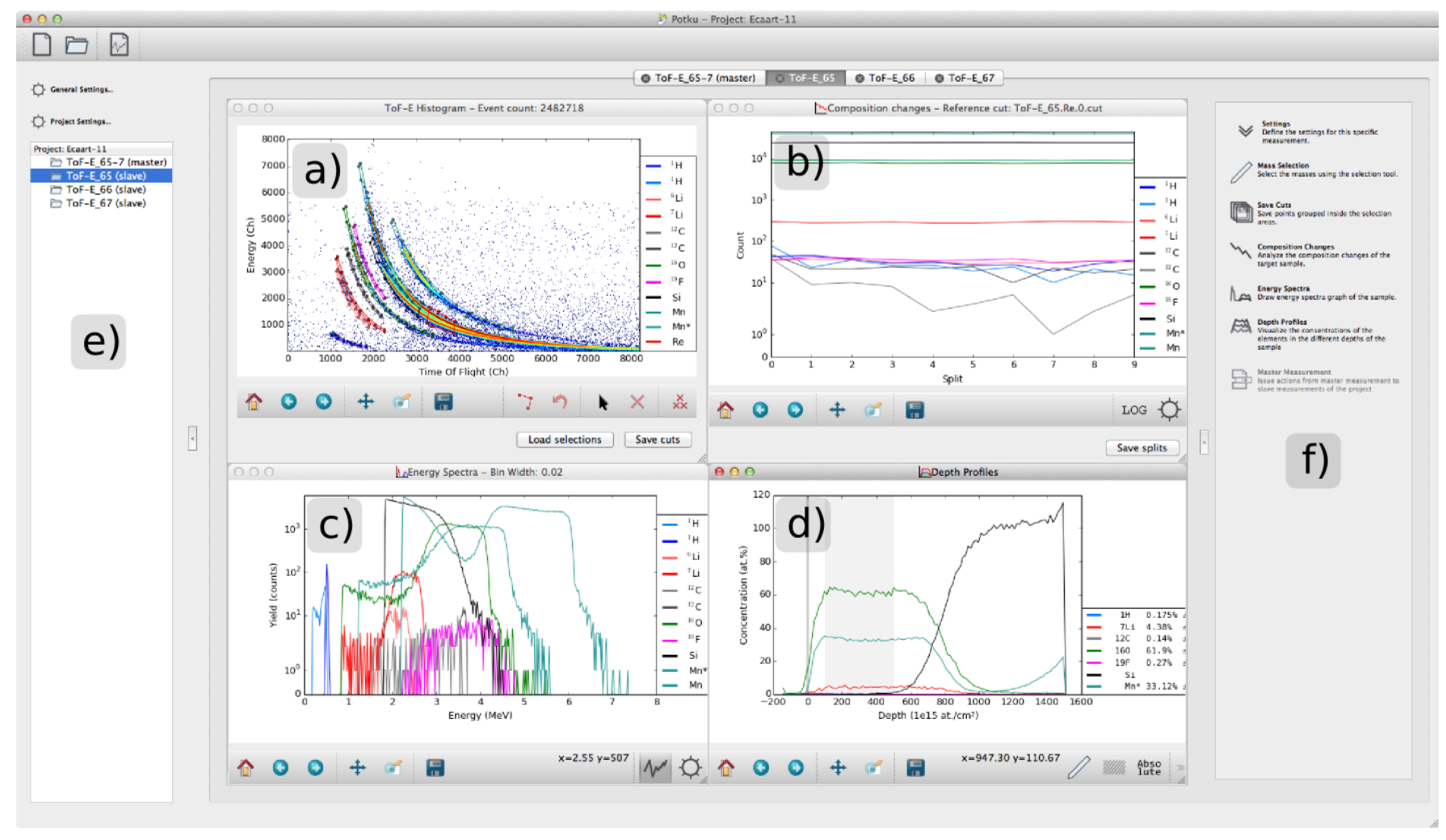

Figure 1: The main window of Potku software. The four subwindows contain a) the ToF-E histogram, b) the composition changes, c) the energy spectra, and d) the depth profiles. The left pane (e) contains dialogs for the general and the project-specific settings, and the list of measurement data in the project. The right pane (f) contains dialogs for different analysis steps.

detector channel as y axis. Intensity is shown as pixel color in a logarithmic scale. Axes can be transposed and inverted and different color schemes can be selected. Since the typical maximum channel number in multiparameter measurements is higher (often $8 \mathrm{k}$ ) than the screen pixel resolution, a compression factor is used to combine several data channels for each display pixel. A default compression factor is set to limit the histogram pixel resolution to about 1000x1000 pixels.

\subsection{Event selection}

The main interactive task in the ToF-ERD analysis is to determine and recognize the different elements and isotopes observed in the data. In Potku this is done by graphically placing polygons around the events in the ToF-E histogram belonging to each isotopic mass (Fig. 2). The user can freely zoom, pan and change the channel compression factors to display the data in the best way when placing the polygon points with a mouse. When the polygon is closed, a dialog appears where the user selects the identity of the element from the periodic table of elements and the isotope of the element from a list of stable isotopes of the chosen element. The user also selects whether the observed element originates from a recoiling (ERD) or a scattering (RBS) process.

Several overlapping polygon selections can be placed for the same element and isotope to study e.g. the surface and the interface contributions of the material.

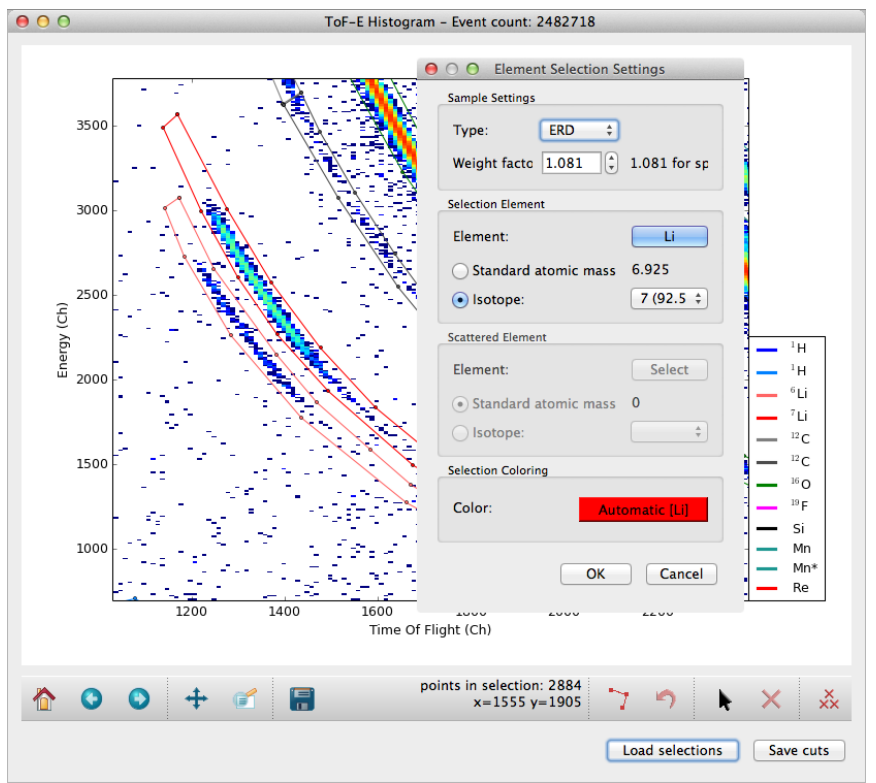

Figure 2: A detail of the ToF-E histogram of measurement data for LiMnO thin-film sample, and the element selection window. The polygon selections for ${ }^{6} \mathrm{Li}$ and ${ }^{7} \mathrm{Li}$ are visible in the histogram. In the elemental selection window the user has recognized the isotope ${ }^{7} \mathrm{Li}$ and set a weight factor to 1.081 to compensate its isotopic abundance. 


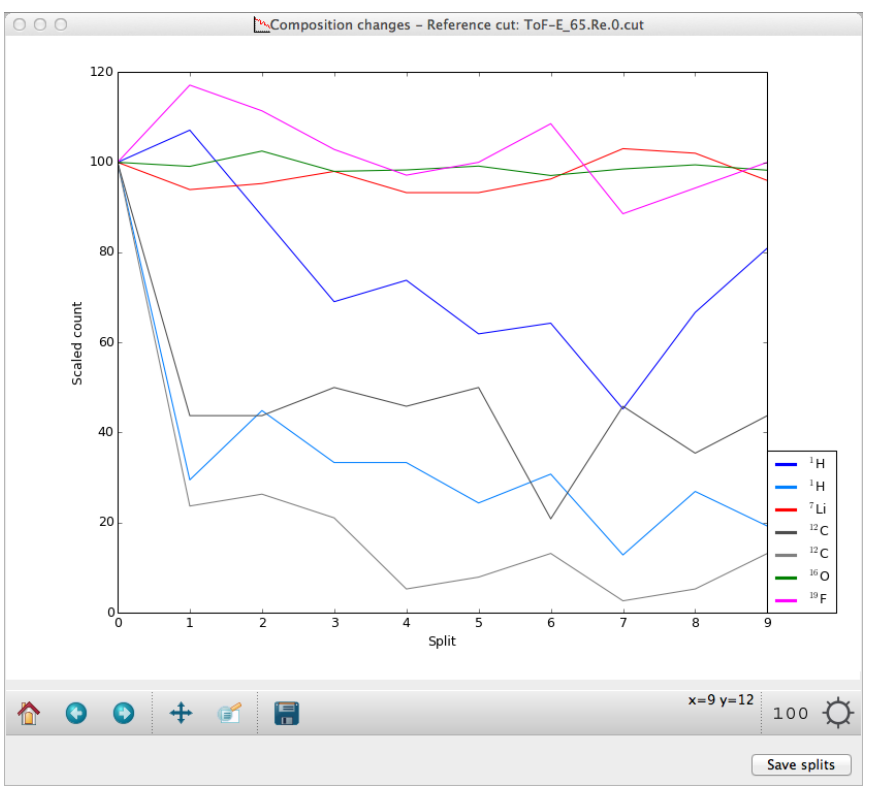

Figure 3: In the composition changes window the measurement has been divided to 10 splits relative to a reference selection from the substrate. Split distributions of individual isotopic components demonstrate how the matrix components $\mathrm{Li}, \mathrm{Mn}, \mathrm{O}$, and the impurity $\mathrm{F}$ remain stable during the measurement but hydrogen and carbon content decrease rapidly in the beginning of the measurement and slower later in the measurement. Separate selections for surface hydrogen and carbon show that the surface contributions of these elements are removed even faster than the corresponding film contributions.

Different isotopes of the same element can either be treated completely separately or, if only one isotope is observed, by using a weight factor which takes into account the contribution of the missing isotopes. Thus, in the case of lithium user can set a weight factor of 1.081 for ${ }^{7} \mathrm{Li}$ instead of selecting both ${ }^{6} \mathrm{Li}$ and ${ }^{7} \mathrm{Li}$ for the analysis.

\subsection{Composition changes}

Data collection in list format allows for straightforward study of composition changes during the measurement without any calibration or measurement setup description. This is done graphically in Potku by selecting a reference data region from the ToF-E histogram representing deep substrate or other contribution where beam-induced changes are not expected (Fig. 3). In the composition changes window the changes of isotopic selections are displayed as a function of split interval compared to the reference selection. For instance, if the number of splits is chosen as 10 , the first value in the figure gives the number of events of each selected element which are located within the first $10 \%$ of the reference data in the original list file.

The composition changes can be displayed in linear and logarithmic scales, and in the scale where the first value of all elemental selections are scaled to 100. The last option usually gives the best representation of beam-induced changes during the measurement. The possibility for making several selections for the same isotope, e.g. representing the surface, the film, and the interface, allows for observing if e.g. hydrogen loss or carbon increase is limited to the surface of the sample.

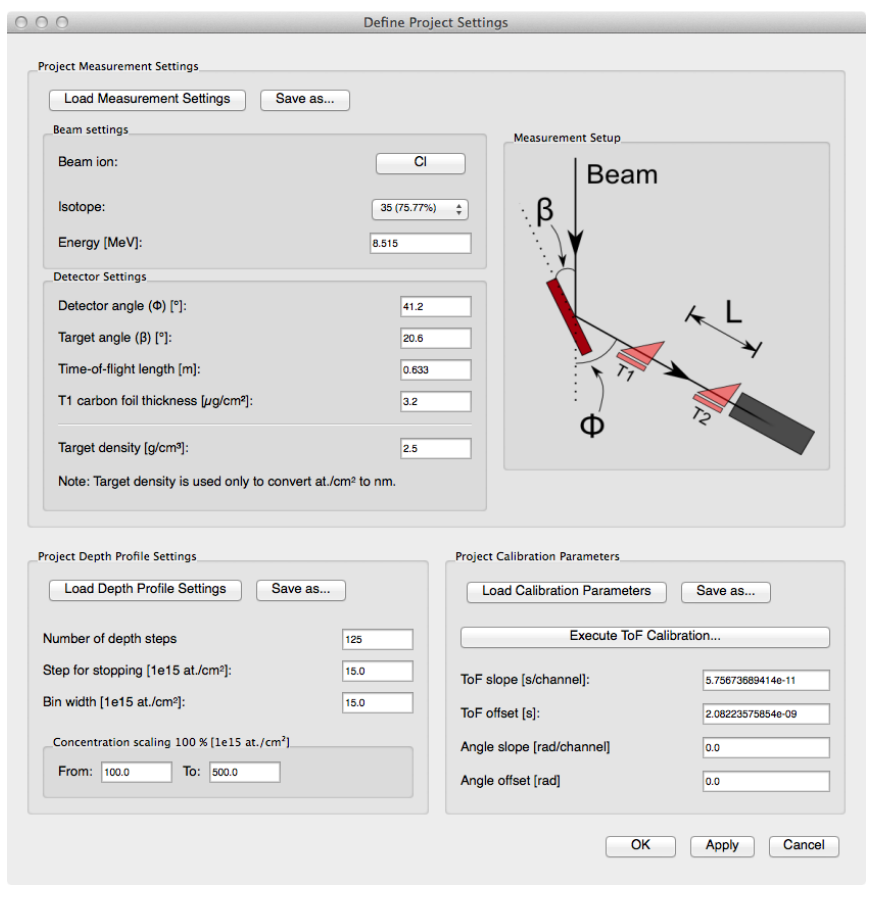

Figure 4: The project settings window.

\subsection{Measurement settings and time-of-fight calibration}

In the measurement settings window the user can set the typical ToF-ERD measurement parameters such as the incident ion, the beam energy, the geometry of the measurement, and the detector parameters (Fig. 4). In the project settings dialog the measurements settings are given for all the measurements in the project. Settings can be changed for individual measurements from the settings dialog in the right pane of the main window. Measurement settings can be loaded from a file generated in an other project in both cases.

Potku software offers a graphical front-end for time-of-flight calibration (Fig. 5). The position of the front edge in the ToF scale for each selection is determined by fitting of an error function to the data. The corresponding time-of-flight value is calculated using the measurement settings and the isotopic identity of the selection and taking into account the energy loss in the carbon foil of the first timing detector. The final ToF calibration is determined by making a linear fit to the edge-channel-flighttime pairs generated in the error-function fits.

\subsection{Energy spectra window}

A depth profile calculation in Potku doesn't require the creation of energy spectra as each energy calibrated event is processed separately in the depth profiling subroutines (Fig. 6). However, the user may want to display and save the energy profile for comparisons with calculations of other software, such as MCERD [11] and SIMNRA. This can be done using the energy spectra feature of Potku software.

\subsection{Depth profile window}

For a depth profile calculation the user selects from a dialog the elemental and isotopic selections which are included in 


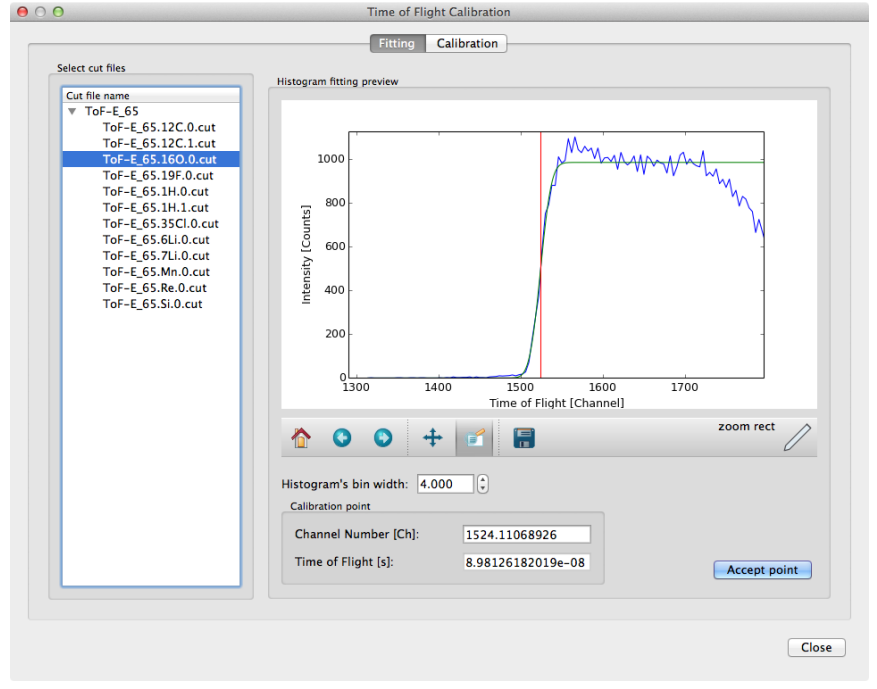

Figure 5: The time-of-flight calibration window. In this case the error-function fit is calculated for the surface-edge of oxygen data.

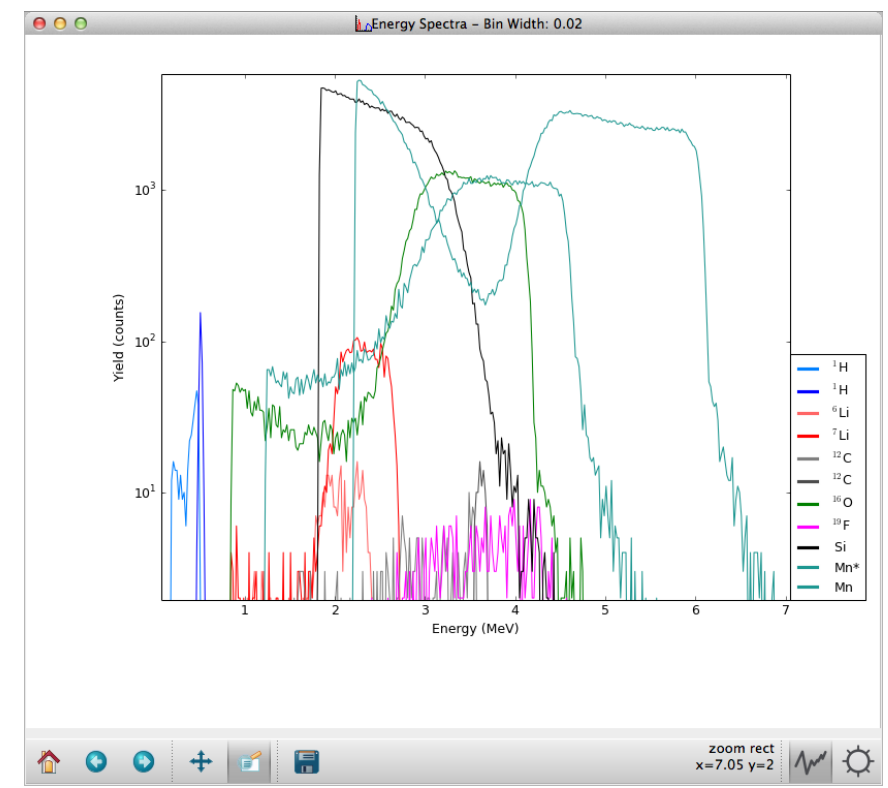

Figure 6: The energy spectra window.

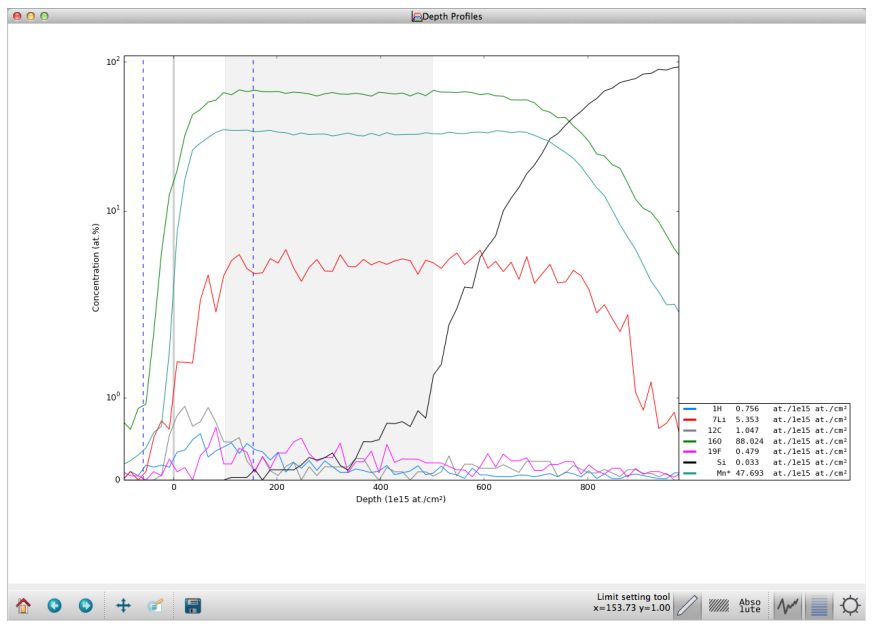

Figure 7: The depth profile window of Potku software. The thin grey line indicates the sample surface. The wide grey region shows the depth range where the total average concentration is set to $100 \%$. The two dashed lines indicate the range where the concentration values are integrated. The absolute concentration values (in the units of $10^{15}$ at. $/ \mathrm{cm}^{2}$ ) are shown in the legend on the right side of the figure.

the depth profile calculation. Then an iterative calculation is performed in a manner described in the section 2.2 using the isotope-specific efficiency files.

The result is diplayed in the depth profile window as a function of either $10^{15}$ at. $/ \mathrm{cm}^{2}$ or, if the sample density is known, in nanometres (Fig. 7). The total concentration of the depth profiles is scaled to the average $100 \%$ within the depth range selected by the user in the measurement settings window. This scaling range and the sample surface are displayed with grey bars in the diagram.

The numeric values of the relative or the absolute concentrations are shown in the legend of the diagram. The values are calculated by integrating the concentration values within a userselected depth range. As the iterative procedure keeps events separated throughout the depth profile calculation the evaluation of the statistical errors is straightforward by counting the number of events for each integrated concentration value. The possibility to exclude elements from the concentration calculation allows the user to avoid e.g. the substrate silicon contribution in the relative composition calculation of a thin film.

In addition to the relative concentrations, the user can display also the absolute concentrations in units of $10^{15} \mathrm{at} . / \mathrm{cm}^{2}$. These values are also integrated from a user-selected range, which allows more complicated manual calculations e.g. for the contribution of the surface hydrogen or interface oxide relative the the corresponding film impurities.

The depth profiles are automatically corrected for angular dependent kinematics, cross sections, and path length effects, if angular data is given in the list mode input. Calibration parameters for the angular data are given in the measurement settings window. An example of the depth profile correction using angular data is given in the Fig. 8. Measurement data of a $\mathrm{Al}_{2} \mathrm{O}_{3} / \mathrm{TiO}_{2}$ nanolaminate sample is used in this example. The sample consist of three $\mathrm{Al}_{2} \mathrm{O}_{3}$ layers and two $\mathrm{TiO}_{2}$ layers, each 


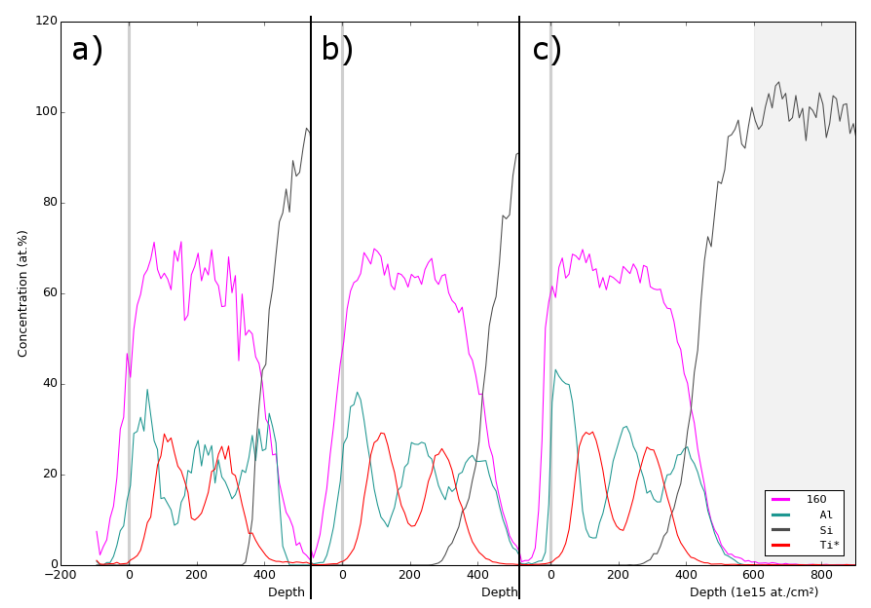

Figure 8: A demonstration of the depth profice correction based on the angular input data. The depth profiles on the left (a) show a result for an analysis of experimental data for a $\mathrm{Al}_{2} \mathrm{O}_{3} / \mathrm{TiO}_{2}$ nanolaminate structure measured with $10 \mathrm{MeV}{ }^{35} \mathrm{Cl}$ beam. The depth profiles in the middle (b) show the corresponding Monte Carlo (MC) simulated depth profiles without the angular correction. The depth profiles on the right (c) are calculated from the MC simulated input data including the angular information.

nominally $10 \mathrm{~nm}$ thick, on silicon substrate. Each element in the structure was simulated with MCERD with the correct relative yields and the resulting data was converted to an integer list mode data file for Potku analysis. Separate data files were generated with and without angular data. The resulting depth profiles generated by Potku demonstrate that the angular correction clearly improves the depth resolution in the first $\mathrm{Al}_{2} \mathrm{O}_{3}$ layer. Below this layer the multiple scattering effects dominate the depth resolution.

\subsection{Automated analysis}

Potku offers a possibility for semi-automated analysis by selecting a master measurement and repeating the identical analysis procedure for the selected slave measurements. A good choise for a master measurement is to combine all the individual measurements files of the project. This allows for creating isotopic selections for the master measurement which cover the regions of all single measurements. Also the low-concentration impurities are easier to observe from the sum file. After performing the initial analysis process according to the master measurement, the single slave measurements can be handled separately by modifying selections, measurement settings and display options wherever necessary.

\section{Future development}

Potku software is used in its current form for commercial ToF-ERD analysis in our laboratory. Improvements and new features are added to the software in regular basis. Short-term development is mainly focused to performance improvements. Currently some of computationally intensive functions, such as selecting the list-mode events within graphically defined polygons, are realized in Python code which reduces the performance of the software. Eventually all the computationally in- tensive subroutines should be re-written to optimize the efficiency.

Graphical selection of elements and isotopes would often be easier if the histogram data would be displayed as ToF-M (mass) or E-M histograms instead of a ToF-E histogram. This conversion would require mass calibration of the measurement data which has been demonstrated in the liteture [12].

\section{Summary}

A software package has been developed for time-of-flight elastic recoil detection (ToF-ERD) analysis. This software, named as Potku, combines a Python-language graphical frontend with $\mathrm{C}$ code computing back-end in a user-friendly way. The software uses a list of coincident time-of-flight-energy events as an input with an optional field for angular data. The ToF calibration can be determined using a simple graphical procedure. The graphical interface allows user to select different elements and isotopes from a ToF-E histogram and to convert the selections to individual energy and depth profiles. Beam induced composition changes can be studied by displaying the event-based data in fractions relative to the substrate reference data. The resulting composition data can be presented as relative or absolute concentrations by integrating the depth profiles within the user-selected ranges. The depth profiles are automatically corrected for the kinematic effects when angular data is given as input. The software is distributed under the GPL license for Linux, Mac, and Windows environments.

Currently the software is adapted only for the analysis of time-of-flight-ERD (ToF-ERD) measurements, but on-going work will later expand the support for gas-ionization detectors and generally for any detection technique which offers elemental separation in ERD or RBS techniques.

\section{References}

[1] M. Mayer, Technical Report IPP9/113, Max-Planck-Institut für Plasmaphysik, Garching, Germany, 1997.

[2] N. Barradas, C. Jeynes, R. Webb, Appl. Phys. Lett. 71 (1997) 291.

[3] J. Maxwell, J. Campbell, W. Teesdale, Nucl. Instrum. Methods B 43 (1989) 218.

[4] F. Schiettekatte, M. Chicoine, S. Gujrathi, P. Wei, K. Oxorn, Nucl. Instrum. Methods B 219-220 (2004) 125.

[5] J. Ziegler, J. Biersack, U. Littmark, The Stopping and Range of Ions in Solids, Pergamon Press, New York, 1984.

[6] J. F. Ziegler, M. Ziegler, J. Biersack, Nucl. Instrum. Methods B 268 (2010) 1818.

[7] W. Bragg, R. Kleeman, Philos. Mag. 10 (1905) 318.

[8] R. Hellborg, H. J. Whitlow, Y. Zhang. (Eds.), Ion Beams in Nanoscience and Technology, Springer-Verlag, Berlin Heidelberg, 2009, p. 179.

[9] H. Andersen, F. Besenbacher, W. Möller, Phys. Rev. A 21 (1980) 1891.

[10] J. L'Ecuyer, J. Davies, N. Matsunami, Nucl. Instr. Meth. 160 (1979) 337.

[11] K. Arstila, T. Sajavaara, J. Keinonen, Nucl. Instrum. Methods B 174 (2001) 163.

[12] M. E. Bouanani, M. Hult, L. Persson, E. Swietlicki, M. Andersson, M. Östling, N. Lundberg, C. Zaring, D. D. Cohen, N. Dytlewski, P. N. Johnston, S. R. Walker, I. F. Bubb, H. J. Whitlow, Nucl. Instrum. and Methods B 94 (1994) 530. 